\title{
VR and AR technologies in the modern cultural space and their role in environmental education
}

\author{
Damira Sirazhiden ${ }^{1, *}$ \\ ${ }^{1}$ L.N. Gumilyov Eurasian National University, 010000, st. K. Munaitpasov 5, Nur-sultan, Kazakhstan
}

\begin{abstract}
Nowadays, in the era of digitalization, it is becoming more and more important to use digital content wisely. This also applies to the cultural heritage of any country. In the context of globalization, reasonable measures should be taken to use content to ensure the sustainability of national identity. In recent decades, new technologies have been developed and used for digital preservation in the form of three-dimensional computer models of various sizes, ranging from very small museum exhibits to the largest cathedrals and castles. The advantages of computer models are undeniable. Through prototyping and reverse engineering, important exhibits are created that make it possible not only to see, but also to hold in one place, thereby allowing a better understanding of historical events and their significance. Three-dimensional visualization provides virtual tours to different places and at different times. Unfortunately, the development of such content is very expensive. Moreover, the technologies for a successful level of immersion are under development. This applies to the criteria for content quality and accessibility and interaction functionality. It is also important to understand what elements to virtualize and how this relates to ensuring cultural identity. Rapidly developing ICT technologies have become a tool for accelerating the creation of information societies around the world. Thanks to this, many traditional services have found a new digital space for functioning and, thus, have reached a global dimension. One example is virtual museums, which provide access to their resources for every interested person who has an Internet connection.
\end{abstract}

\section{Introduction}

Virtual reality (VR) is defined as the development of a simulated experience that is somewhat similar to a real-time situation. In 1994, the first virtual reality-based modeling language was first designed, which was used to humanize the virtual world to overcome the craving for headsets. This technology offers a wide range of applications, namely: medical training, video games, military goals, etc. Moreover, virtual reality allows you to immerse yourself in the world of culture and art without taking into account any borders, while staying up to date with innovations developing in this direction [1].

\footnotetext{
${ }^{*}$ Corresponding author: kostya15.00@mail.ru
} 
Virtual reality (VR) is a technology that is based on feedback between a person and the world synthesized by a computer, as well as the way in which a person visualizes the digital world, manipulates it, and interacts with it.

The definition of augmented reality (AR) has also been introduced recently. The term was originally invented by researcher Tom Codel in 1990, who was working for Boeing at the time. In 1997. Ronald T. Azuma, in his research on various ways to use augmented reality, gave it a rather capacious and simple definition - it is a system that: combines the virtual and the real, interacts in real time; $\square$ is located in three-dimensional space. Augmented reality, according to Azuma, is a kind of virtual reality, but with one clarification: AR integrates and complements the real world instead of completely replacing it, as VR does [2].

At the present stage, especially in the context of the COVID-19 pandemic, it is not possible to establish many cultural links in real time in order to avoid the mass spread of infection. For this reason, VR and AR technologies in the field of culture today allow not only to expand the horizons of people who are not able to regularly keep up with the news of culture and art due to their lack of access to the cultural treasures of the world, but also serve a continuous process of cultural penetration into everyday life, without creating a danger to human health. In particular, now any inhabitant of the planet, through the use of VR and AR technologies, can safely visit the desired museums and exhibitions, concerts, vernissage and replenish their cultural knowledge with the necessary information [3].

Every year, VR and AR technologies are improved, which allows you to expand the range of cultural services that they can help provide.

\section{Materials and methods}

The paper uses analytical and synthetic methods used to assess the role of VR and AR technologies in the development of modern culture. The possibilities of using VR and AR technologies to increase the role of cultural heritage in society are investigated.

\section{Results}

The role of virtual reality and augmented reality (VR/AR) technologies in people's lives is growing day by day. Many people are familiar with the term virtual reality, but are not sure about using this technology. Games are an obvious virtual reality application, just like virtual worlds, but there are a number of uses for virtual reality-some of which are more complex or unusual than others. VR/AR technologies are already widely used in the military, education, healthcare, entertainment, fashion, tourism, and heritage, business, engineering, sports, media, scientific visualization, telecommunications, construction, etc. Now opportunities and innovations are emerging in other sectors related to the use of VR/AR [1]. As for cultural heritage use cases, this applies to the use of VR/AR in museum and historical settings, such as in visitor centers. These settings use interaction as a means of communicating information to the general public in new and exciting ways [4].

Today, there is a withdrawal from the traditional type of experience associated with museums, galleries, and visitor centers. The old model was passive engagement, in which people viewed exhibits but did not engage in experiences where interaction is the main feature. Interactive displays make up a significant part of many exhibitions and are particularly attractive to children. Children are often difficult to attract to a museum or gallery, as they tend to view it as a boring experience. But the use of interactive technologies such as virtual reality has changed this perception and opened up these spaces to a new audience [5]. 
Culture, heritage and art have attracted people to tourist destinations for a long time. This trend is reflected in the growing number of tourists who are looking for adventure, culture, history, archeology and communication with the local people. Cultural heritage and tourism are important for a number of countries, they have a positive economic and social impact, establish and strengthen identity, and so on.

The use of VR and AR technologies in the modern cultural space will solve a number of interrelated tasks:

- improving the way of delivering cultural heritage content with a sustainable approach;

- engaging the public in an entertaining and active way of understanding the significance of cultural heritage sites;

- increasing the depth of immersion for professionals and ordinary users in different historical periods [6].

Researchers note that in the modern cultural space, the use of virtual and augmented reality technologies should be based on gamification scenarios. There are augmented reality (AR) solutions for mobile devices that help users find information about various cultural sites. Using a smartphone's video camera, screen, and internal sensors, real-time video frames are combined with two-dimensional data such as images and text descriptions. However, this is just an informational search and image, without the involvement of participants and motivation to actually find a real place in nature. This is why gamification scenarios that integrate the use of mobile devices and other gadgets for the general public play an important role in popularizing the cultural realities of different eras [7].

In addition to interacting and solving scenario tasks, you need to use not only twodimensional extensions (photos and text), but also 3D (three-dimensional) extensions, which include static 3D models of ancient monuments and dynamic animated 3D models. For local VR (virtual reality), you can use passive HMD frames in combination with smartphones [8].

The content of virtual reality scenarios can be based on 360-degree photos and videos with active fields for quizzes and tests. Physical architecture should be placed on mobile solutions, which will expand the audience of people who want to get acquainted with the cultural heritage.

One of the ways to implement these technologies is through interactive exhibitions that are organized and held in a virtual space. Some of these exhibitions offer dynamic cybervisits. So, in the Exploratorium San Francisco tere is an extensive memory exhibit that uses approaches as diverse as dissecting a sheep's brain and studying the importance of hair in facial recognition. Some exhibits are unique to the website, while others are digital versions of real exhibits Exploratorium, for example, a guide to Drosophila mutants for beginners [6].

Online exhibitions on the website of the University of California Museum of Paleontology are also of interest. For example, "Introduction to phylogeny" teaches viewers how to construct a cladogram based on common derived characteristics of organisms. The convergent evolution of vertebrate flight, which has evolved three times over 500 million years of vertebrate history, is also widely discussed here.

Those who want to explain or understand the genetic code will find a lot of information on the website of the Technical Museum of Innovation. After descriptions of genome mapping and scientists' hopes for a cure for genetic diseases, a reality check follows, since the site presents hypothetical ethical dilemmas arising from genetic knowledge [7].

The AnthroNews section of the American Museum of natural history website includes exhibits (such as three-dimensional images of human skulls), discussions (such as the origin of modern humans), and features (such as human aging).

The most successful online museums are those that use the dynamic potential of the Internet to offer exhibits that work just as well both online and in real life. At the Institute 
and Museum of the history of science in Florence, you can take a virtual tour of the Galileo room in the museum.

The busiest online museum is London Natural History Museum. Using suitable virtual reality software, you can participate in the Virtual Endeavour exhibition, an experiment in a new virtual exhibition environment [9].

To date, it can also be argued that VR and AR technologies have allowed the inhabitants of the planet not to stay in a closed space, but to continue active communication in the cultural space. One of the areas of application of these technologies is the museum sphere [3].

One of the electronic services is digital museums, the most effective element of which is a virtual tour. Undoubtedly, this service deserves to be called a promoter of culture, which is particularly effectively manifested during the period of international quarantine measures. In this regard, it is important that the virtualization of museum resources can be constantly monitored and supported, not only financially, but also, for example, by analyzing and identifying areas that require special attention.

In a world of widespread information dominance, the role and tasks of museums have expanded, as previously unavailable opportunities for registering, archiving and promoting collections in digitized form have appeared [7].

Moreover, it was also observed:"...creative activity is the creation of culture, reality, and sometimes art, implemented in the digital virtual sphere"[1]. The introduction of ICT in the museum sector has made monuments and exhibits a tourist attraction accessible to all [8].

Virtual museum tours have many advantages. The most important of them from an economic and social point of view are [9]:

- dissemination of new technologies, creation of new goods and services, as well as support for creativity;

- international branding and increased territorial attractiveness;

- economic diversification, new jobs and income through the development of cultural and creative neighborhoods;

- improving the quality of life;

- higher level of social capital;

- knowledge development and professional development;

- increasing the number of culturally aware and open communities;

- the proliferation of creativity;

- the role of the partner in the educational process [10].

Museums must accept the challenge of spreading knowledge in the virtual space and see it as a mission in which the reward is the formation of identity and the formation of communities not only in the region, but also around the world.

However, the advantages associated with the development of virtual walks and museum excursions are much broader and more versatile. For example, they relate to marketing, financial, and social aspects. These e-services are also characterized by relatively low production costs and preparation time. From a technical point of view, this is not a very complex process, and currently there are many companies on the market that provide services for creating such applications. Taking into account the above saying, as well as the fact that quarantine contributes to increased interest in electronic services, remote work and electronic entertainment, it can be argued that during the quarantine period caused by the SARS-CoV-2 coronavirus pandemic, museums need to actively and dynamically develop virtual walking services.

To prove the above, we present a comparison of the results of research conducted in August 2019 and April 2020 on the development of services that involve virtual walks through monuments and Museum centers located in Poland. 


\section{Discussion}

In August 2019, researchers conducted the first study of virtual tours of Polish museums. 112 digital tours were analyzed, 102 of which were successfully launched [2].

The following analysis parameters were used:

- the amount of content included in the service and covering all types of materials: panoramas, photos of objects, $3 \mathrm{~d}$ photos, descriptions, etc.;

- convenience for visitors with disabilities (especially for the visually impaired and hard of hearing), for example, ease of perception of the lecturer, speaker;

- the presence of voice control, the ability to enable subtitles or change the font size;

- ability to implement additional options;

- ability to perform actions in virtual space;

- the level of functionality that allows visitors to move around during a virtual walk;

- smooth and trouble-free operation of the app, fast movement between locations and fast loading of content;

- additional sounds, music, postcard creation, VR option;

- quality and graphic appeal;

-level of freedom of movement in virtual space;

- the ability to choose a foreign language and the level of its use in various functionality, options, and digital content [2].

These parameters were evaluated using a point system from 1 to 5 .

A similar study was conducted for the same parameters in the period from April 6 to April 20, 2020.

The results of the analysis showed the following. Both during the immediate period of the pandemic and in the period preceding it, a low average score was obtained for the following parameters -

- convenience for visitors with disabilities (especially for the visually impaired and hard of hearing), for example, ease of perception of the lecturer, speaker - 2.2 points;

- ability to implement additional options - 1.4 points;

- ability to perform actions in virtual space -1.3 points;

- smooth and trouble-free operation of the app, speed of movement between locations and fast loading of content-1.3 points;

- additional sounds, music, postcard creation, VR option -2.1 points;

- the level of freedom of movement in virtual space is 1.9 points [2].

A survey of potential users of these virtual systems, as well as an analysis of the number of requests to them, showed that during the pandemic, interest in these applications increased several times. However, many of them did not justify the trust of users, which restricted access to museum archives and exhibits in Poland. The final assessment of the level of development of virtual museum tours in Poland suggests that it is quite low.

Among the reasons that reduce the level of implementation of these technologies in cultural institutions in general and in museums in particular, we should note the lack of desire among the museum's management and employees to implement these projects, as well as to improve their professional skills, taking into account the Informatization and automation of the museum environment. This factor significantly hinders the ability of museums both in the field of popularization of Museum collections and in economic terms, since during the period of restrictions on the ability of people to move freely, there is a decrease in financial revenues from ticket sales at museum ticket offices. The introduction of VR and AR technologies will not only expand the horizons of virtual museum visitors, but also allow museums themselves to maintain a balance of financial revenues, which is 
directly related to the expansion of Museum funds and replenishment of museum collections, which is also very important for the development of the cultural horizons of present and future generations.

\section{Conclusions}

To increase the efficiency of implementing VR and AR technologies, you need the following:

- it is necessary to increase the level of information literacy and awareness of museum staff;

- museums should apply the principle of "good practice", and not be modeled on lowquality services provided by other institutions in the region;

- the percentage of remote employees during the pandemic should be increased;

- cooperation with the social sector should be expanded, for example, by creating digital communities gathered around the museum, its collections and history [3]. This will allow you to get interesting ideas, support it work on a voluntary basis, and get more collections (for example, in digital form) from local collectors;

- the museum service must have constant informational, content and management support that will ensure its proper functioning, expansion and attractive advertising of the Museum (the success of the service can only be achieved through interdisciplinary cooperation [8]);

- museums should consider the development of electronic services related to virtualization as a great opportunity to promote cultural heritage [5];

- the approach of creating virtual walks should not be "universal" - it should be original and innovative;

- lack of collection can be compensated by other features such as more detailed presentation, adding a visit the building inside and out, the addition of the ability to walk at different times of year and day, etc.;

- virtual tour should be constructed in such a way that the visitor has the impression of the greatest possible freedom and openness, as well as a large number of different types of interactions - only then it can compete with other services that provide access to the virtual space (e.g., computer games);

- the potential and resources of museums should be fully used. A single service should combine content in various forms, such as panoramas, photos of 3D objects, movies, sounds, and music;

- creative tools in the application, with the help of which operate from a virtual path must be fully used;

- the availability of the service should be improved by translating content into at least English, and the number of facilities for users with disabilities should be increased.

\section{References}

1. Lisbon European Council, 23 And 24 March 2000, access: 18 April 2020, https://www.europarl.europa.eu/summits/lis1_en.htm.

2. P. Gutowski, Development of Virtual Museums in Poland, Studia Periegetica 3 (27), 9 (2019)

3. A. Lundgren, E.H. Lundevaller, Micro-simulation modelling of domestic tourism travel patterns in Sweden Education (2004) 
4. Museums and Local Development in Poland, OECD Local Economic and Employment Development (LEED), project led by E. Travkina, research team led by M. MurzynKupisz (2019)

5. A. Vahtra-Hellat, Coronavirus emphasises the necessity of e-services (2020), https://ega.ee/blog_post/coronavirus-emphasises-the-necessity-of-e-services/.

6. D. Lacitignola, I. Petrosillo, M. Cataldi, G. Zurlini, Modelling socio-ecological tourism-based systems for sustainability, Ecological Modelling $206(1), 191-$ 204 (2007)

7. Heritage authenticities - A case study of authenticity perceptions at a Danish heritage site, Journal of Heritage Tourism 9 (4), 285-298 (2014)

8. S. Minton, State of the Market: Global IT Spending Review and Outlook April 2020 (IDC - Analize the Future, 2020)

9. Towards 2020 The British Museum's Strategy (2020) (published by British Museum, 2020), https://www.britishmuseum.org/sites/default/files/2019-10/Towards_2020The_British_Museum_Strategy.pdf.

10. A. King, (The impact of COVID-19 on user behaviour and ecommerce, 2020) https://www.ayima.com/blog/the-impact-of-covid-19-on-user-behaviour-andecommerce.html.

11. N. Metzger, R.N. Zare, Interdisciplinary research: From belief to reality Science 283 (5402), 642-643 (2001)

12. D. Rhoten, Interdisciplinary research: Trend or transition, Items and Issues 5 (1-2), 611 (2004)

13. The Biggest Business Impacts of the Coronavirus Pandemic, eMarketer and BII analysts identify what to watch for in tech, digital marketing, banking and digital health (2020), https://www.emarketer.com/content/the-biggest-business-impacts-ofthe-coronavirus-pandemic-according-to-business-insider-intelligence.

14. W.van der Weiden, (2012), Virtual Museums: from opportunity and threat to chance and challenge (The Learning Museum. Report 1 - The Virtual Museum, 2012) 